\title{
Research on smart manufacturing execution system (Smart-MES) for modern numerical control shop floor: functional components and its implementation strategies
}

\author{
Ming Huang, Qi-sen Zhou, Ao Bai \\ Institute of Machinery Manufacturing Technology, China Academy of Engineering Physics, China \\ baiao1983@126.com
}

Keywords: manufacturing execution system; smart factory; lean manufacturing; intelligent manufacturing; production management; machining workshop; function model; implementation method

\begin{abstract}
Manufacturing Execution System (MES) is the core application platform of intelligent manufacturing for the modern Numerical Control (NC) shop floor under the background of industry 4.0. Based on the characteristics and the business model of production operation in the modern NC shop floor, the system process of MES is analyzed in detail, then the function modules are proposed and built, and the strategies of rapid process design, production planning and scheduling, visualized monitoring of manufacturing process, manufacturing field data collection, online quality monitoring, man hour quota management, material management and were determined and established. The proposed manufacturing execution system is a collaborative management information platform integrating process design stage, production preparation stage, and production execution stage, whose feasibility and effectiveness have been proved in a modern NC shop floor.
\end{abstract}

\section{Introduction}

Nowadays, high efficiency, high quality and low cost are the most important goals for modern manufacturing enterprises. Manufacturing execution system (MES) are computerized systems widely used in manufacturing, to track and document the transformation of raw materials to finished goods. MES provides information that helps manufacturing decision makers understand how current conditions on the plant floor can be optimized to improve production output[1]. In a word, MES is an effective tool and weapons to realize the goals above for manufacturing enterprises to maintain their competitiveness in the global market. According to raw data collection and production information share, various problems encountered everyday in the manufacturing field of shop floor can be solved, and the lean management of production activities from production preparation (including production plan, process design, quota making, et al.) to production execution (including task management, production progress tracking, process quality management, material delivery, inventory control, et al.) can be also easily achieved. In literature [2], MES architecture for FMS compatible to ERP (enterprise planning system) is built, and the proposed MES is demonstrated by applying it to two FMS (Flexible Manufacturing Line) lines. In literature [3], a distributed object-oriented approach to develop a system framework for the computer-integrated manufacturing execution system is proposed, and a variety of functional components by inheriting appropriate design patterns of the Framework is designed. In literature [4], a method to align a manufacturing execution system with Lean objectives is proposed. Literature [5] explores the factors that affect the successful implementation of the Manufacturing Execution Systems (MES) in small and medium manufacturing enterprises.

Different manufacturing enterprises and production mode need different methods to adopt and implement MES based on management requirement of shop floor and feature of product. In this study, a smart manufacturing execution system (Smart-MES) for modern numerical control shop floor is proposed. Based on the business process analysis, the functional components and implementation strategies of MES is given out and illustrated in detail. 


\section{Business Process Analysis}

In modern NC shop floor, the business process is generally run as follows. First, customers' requirements or orders from external market are converted into master production plans of factory, and the production planners arranges production preparation (including process design and quota formulation) and production execution (production execution plan for final products, components and parts) according to production plans. Then, the different work team receives the production tasks, prepare raw materials and dispatch the tasks to workers or operator. In the production process, production process information and production quality information are recorded by the dispatcher and quality inspectors. Finally, the finished products which have passed the examinations are put into storage and then delivery to customers who needs. The general production business process of modern NC shop floor is depicted in figure 1.

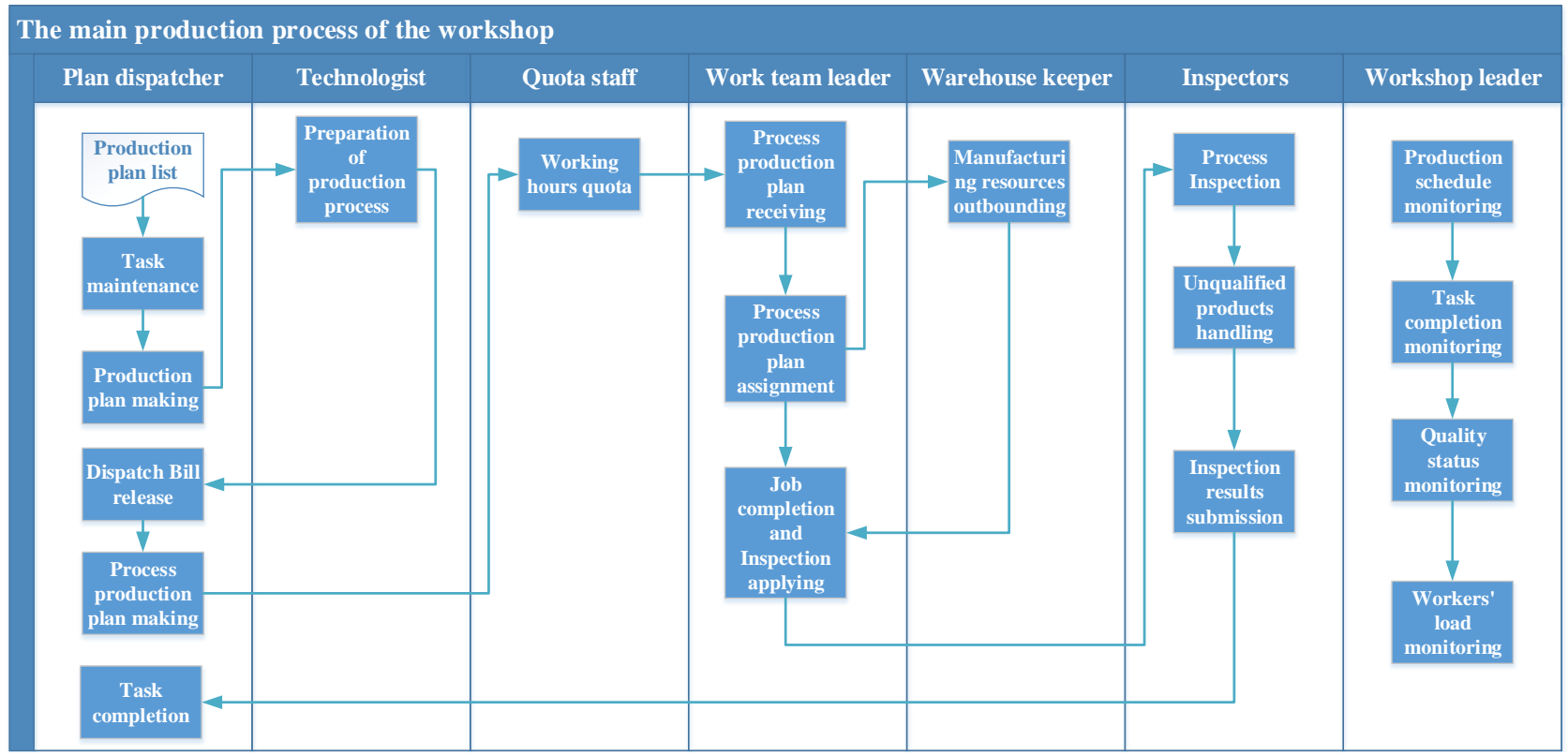

Fig. 1 General production business process of modern NC shop floor

\section{Function Module of MES}

Based on the business process of Modern NC shop floor, the function module of MES will be given out and described in detail in this section. The overall function of the MES consists of two parts which are business function modules and system function modules. Furthermore, the business function modules are mainly divided into four types of modules, namely, Production Preparation Module (PPM), Manufacturing Field Management Module (MFMM), Resource Warehouse Management Module (RWMM) and Data Statistical Analysis Module (DSAM). The system function modules mainly provide users information management and rights management. The detail of the MES functions are just depicted in figure 2 and described as follows. 


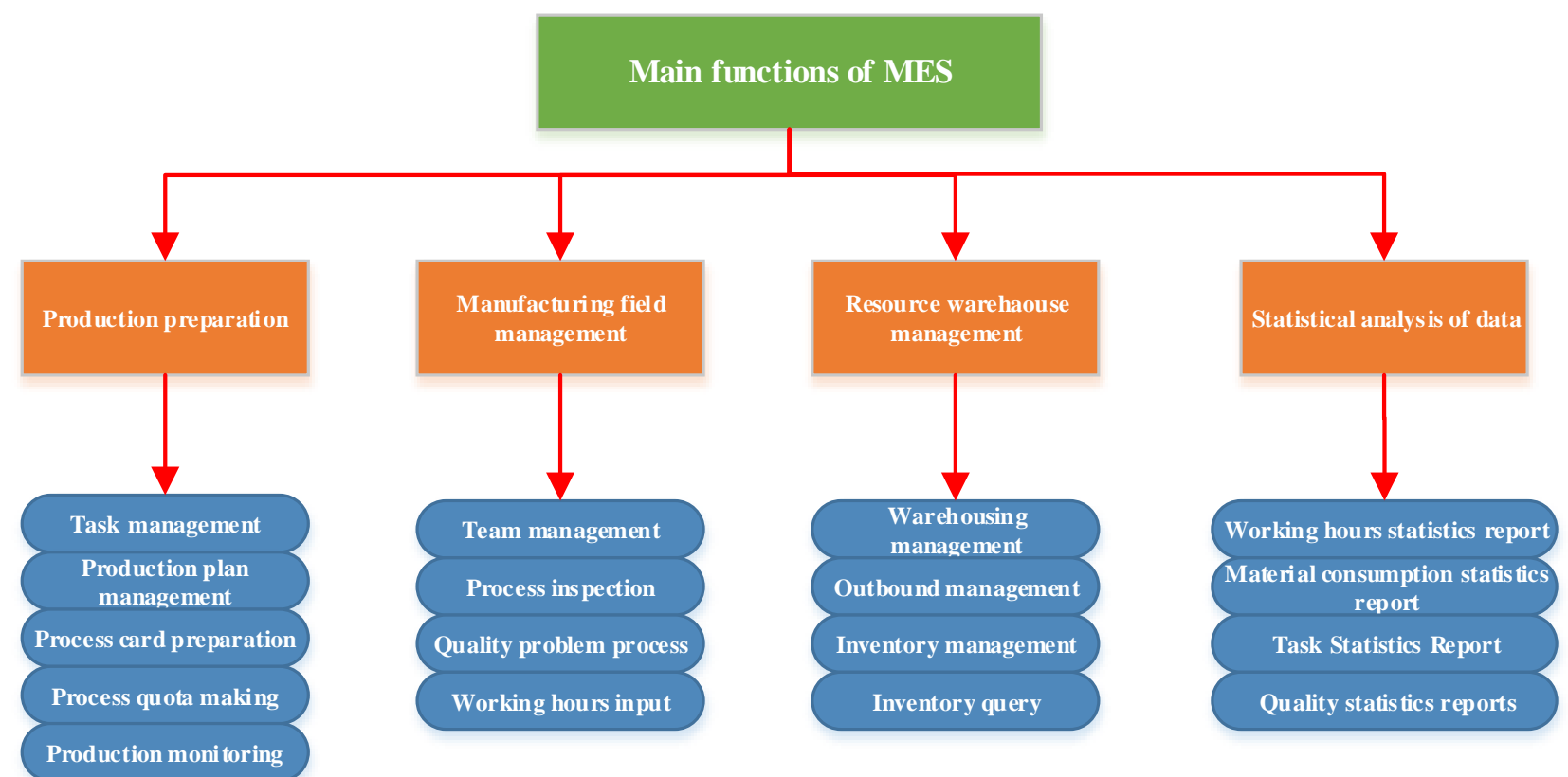

\section{Fig. 2 Function module of lean manufacturing execution system Production Preparation Module (PPM)}

Production preparation is an important task for discrete manufacturing enterprise, which guarantees that the production task can be executed smoothly in shop floor. Production Preparation Module includes five sub-function modules, namely, task management, production plan management, process card preparation, process quota making or formulation and production progress monitoring. Task management is to input production orders or production tasks information into MES, the orders/tasks information can be manually established and maintained, also, it can be imported quickly according to electronic file in excel format. Production plan management is to make and develop the different levels of production plan of final products, components, parts, et al. Based on the equipments' real-time capacity load and production tasks' priorities, the system can achieve computer-aided production scheduling, which will arrange task in a more scientific and reasonable manner to ensure that the production tasks can be delivered on time. Process card preparation is used to make production route based on design requirements and technical requirements. Through the process of preparation, the processing equipment, craft equipment and cutting tools can be determined, and the quality inspection specification can be also given out base on the product design requirements. Process quota formulation is used to determine the standard operating time for each process, the quota (also called work hour) can measure the workload of workers on the one hand, and on the other hand provides time scale for production schedules. The function of production monitoring can trace the production execution progress in a real-time manner, therefore the production task arrangement can be adjusted to adapt the changes of production status or any emergencies.

\section{Manufacturing Field Management Module (MFMM)}

Manufacturing Field Management Module includes four sub-function modules, namely, team management, process inspection, quality problem processing and working hour input. With team management module, the team leader accepts the production tasks from their personal computers, and then assigns them to the operators considering their workloads and operation skills (experience or knowledge). After the process task finished, the parts of work-in-process will be sent to quality inspection section or department to have an examination if necessary, and the inspection result will be input into the system. In the quality inspection process, when product quality problem occurs, a quality problem processing process will be launched, different job roles (such as designer, technician, worker, leader, et al.) will be involved to deal with these problems and clarify the corresponding responsibility. After the completion of production and quality inspection, the working hours will be 
gathered and input into the system's database, which will measure and evaluate the workload of workers quantitatively.

\section{Resource Warehouse Management Module (RWMM)}

Resource Warehouse Management Module includes five sub-functional modules, namely, warehouse management, outbound management, inventory management and inventory inquiry. Warehouse management is a very important work to ensure that the manufacturing resource or material can satisfy the production's needs or requirements. By warehouse management module, it will efficiently realize the warehouse management of resource and materials. Outbound management can realize the outbound operation and monitor the consumption of resource and materials. Inventory management and inventory inquiry module can easily check and count the change and usage of inventory, thus achieve lean management and control of manufacturing resource.

\section{Data Statistical Analysis Module (DSAM)}

Based on real-time manufacturing field data (such as product data, task data, work hour data, quality data et al.) collection and analysis, Data Statistical Analysis Module can provide a wide range of reports, including work hour statistics reports, material consumption reports, production progress reports, production task reports, quality statistics reports. Of course, user can also obtain user-defined report if need. With this different types of reports, production manager or leader can make quickly and scientific decision, which will greatly promote the lean management of NC job shop, and provide foundation for Intelligent Manufacturing (IM) in the near future.

\section{Implementation Strategies and roadmaps of MES}

In order to guarantee the success rate of MES implementation and control failure risk in NC job shop, it is very necessary to follow several principles of implementation steps and methods. The principles can be summarized in short term such as system planning, focusing on the application, small step and fast run, emphasizing effectiveness, controlling risk, et al. In the MES implementation of the full life cycle, it is necessary to clarify the deliverables and major outputs at each stage, ensure the quality of the system execution, and effectively control the construction progress and risks. In addition, the implementation of MES should also pay special attention to the following three aspects:

1) Through the analysis and organization of the production process, a more scientific and effective integrated production management system and architecture should be established, and the production business process of the NC job shop should be built, the business model of the workshop should be also determined.

2) Different types of business forms and reports (such as production task bill, production process card, quality inspection record, unqualified product processing sheet, et al.) which are used commonly in daily work should be pay special attention to. Production related basic data must be collected and standardized, thus the data model of the NC workshop can be built.

3) A joint project implementation team is also necessary. On the basis of the MES implementation goal and work tasks, the responsibilities of the stakeholders should be clearly defined and segmented. Defining the MES's requirements and their priorities, thus to ensure that the core and key requirements can be firstly satisfied.

The general implementation steps of MES in modern NC shop floor are just depicted in figure 3. 
- Training the key users, and the users are familiar with the system operation process

- Through the execution of the actual typical tasks to verify the operation performance of the MES system

- The user can complete the production business

officially process through the system

- The improvements and optimization of system's functions and user's graphics interface

Fig. 3 Implementation steps of manufacturing execution system in NC shop floor

\section{Conclusions}

Base on the manufacturing filed data collection and analysis, manufacturing Execution System can not only realize the lean management of the whole production process of the NC job shop, but also provide decision support to promote the ability for customers' orders delivery. It is believed that the MES will play a more and more important role in production innovation and reform for vast of China's small and medium-sized manufacturing enterprises, and lay the foundation for intelligent manufacturing implementation under the background of Industry 4.0 and industrial internet.

\section{Acknowledgements}

This work was financially supported by the Informatization Special Research Project of China Academy of Engineering Physics (CAEP) (Contract No: K1078-16-XX).

\section{References}

[1] Information on https://en.wikipedia.org/wiki/Manufacturing_execution_system.

[2] B.K. Choi and B.H. Kim: Int. J. Comput. Integ. Manufacturing Vol. 15(2002), p. 274-284.

[3] F. T. Cheng, E. Shen, J. Y. Deng and K. Nguyen: Int. J. Comput. Integ. Manufacturing Vol. 12(1999), p. 384-402.

[4] J. Cottyn, H. V. Landeghem, K. Stockman and S. Derammelaere: Int. J. Prod. Research Vol. 49(2011), p.4397-4413.

[5] S. M. Lee, S. G. Hong, P.Katerattanakul and N. R. Kim: Int. J. Prod. Research Vol.50(2012), p.1942-1954. 